\title{
Evaluación positiva de medicamentos: junio/julio 2013
}

\author{
Álvarez Herranz P. ${ }^{1}$, Prats Olivan P. ${ }^{1}$, Puerro Vicente M. ${ }^{2}$, García Luque A. ${ }^{3}$ \\ Sanid. mil. 2013; 69 (4): 260-265; ISSN: 1887-8571
}

\section{RESUMEN}

Se reseñan los medicamentos ya evaluados por la Agencia Española de Medicamentos y Productos Sanitarios hechos públicos en los meses de junio y julio de 2013, y considerados de mayor interés para el profesional sanitario en el ámbito hospitalario. Se trata de opiniones técnicas positivas que son previas a la autorización y puesta en el mercado del medicamento.

Positive assessment of drugs: June/July 2013

SUMMARY: The drugs assessed by the Spanish Agency for Medicines and Health Products made public in June and July of 2013, and considered of interest in hospital healthcare professional, are reviewed. These are positive technical reports prior to the authorization and placing on the market of the product.

\section{ALEMTUZUMAB (LEMTRADA®) $)^{1-5}$}

Este medicamento está indicado en el tratamiento de pacientes adultos con esclerosis múltiple remitente recidivante, con enfermedad activa definida en base a criterios clínicos o de imagen.

El principio activo del medicamento, el alentuzumab, es un anticuerpo monoclonal humanizado, dirigido contra la glicoproteína de superficie celular CD52. No se ha demostrado el mecanismo de acción por el cual se ejercen los efectos frente a la esclerosis múltiple, pero las investigaciones realizadas sugieren que se producen los efectos inmunomoduladores a través de la depleción y repoblación de linfocitos.

Los estudios clínicos efectuados, en los que se compara la actividad de Alemtuzumab frente a Interferón beta 1a, muestran que el tratamiento con alemtuzumab es seguro y eficaz, demostrado una reducción de la tasa de recaídas y en algunos de los ensayos clínicos se ha observado una ralentización en la progresión de la discapacidad.

Los efectos secundarios manifestados con mayor frecuencia en la fase de ensayo clínico han sido: reacciones asociadas a la perfusión (dolor de cabeza, enrojecimiento facial, náuseas, urticaria, erupción, prurito, fiebre y cansancio), infección del tracto respiratorio superior, infección del tracto urinario, linfopenia y leucopenia y alteraciones de la glándula tiroides (hipotiroidismo, hipertiroidismo, bocio y alteraciones autoinmunes).

\section{AFATANIB (GIOTRIF®) $)^{6-8}$}

El afatanib es un inhibidor de la proteín kinasa a la que se covalentemente bloqueando de forma irreversible al seña-

\footnotetext{
Cte. Farmacéutico. Servicio de Farmacia Hospitalaria

2 Tcol. Médico. Servicio de Farmacología Clínica.

3 Cte. Médico. Servicio de Farmacología Clínica.

Hospital Central de la Defensa Gómez Ulla. Madrid. España.
}

Dirección para correspondencia: Servicio de Farmacia Hospitalaria. Hospital Central de la Defensa Gómez Ulla. Glorieta del Ejército. 28047 Madrid. España. palvher@oc.mde.es

Recibido: 2 de octubre de 2013

Aceptado: 14 de octubre de 2013 lización de todos los homo y heterodímeros formados por los miembros de la familia de factores de crecimiento ErbB: EGFR (ErbB1), HER2 (ErbB2), ErbB3 y ErbB4.

Este efecto hace que el medicamento Giotrif esté indicado en el tratamiento en monoterapia de pacientes adultos con cáncer de pulmón no microcítico localmente avanzado o metastásico, con mutaciones activadoras del receptor del factor de crecimiento epidérmico (EGFR) que no hayan sido tratados previamente con un inhibidor de la tirosina quinasa del EGFR.

La autorización de este medicamento se basa en distintos ensayos clínicos. Uno de ellos, en el que se compara afatinib frente a placebo en pacientes con carcinoma de pulmón no microcítico, donde afatinib muestra una mejoría de los síntomas y un retraso en el tiempo de progresión de la enfermedad. Otro de los ensayos en fase III, aleatorizado y abierto de afatinib frente a quimioterapia en pacientes con cáncer de pulmón no microcítico localmente avanzado o metastásico y con activación de la mutación EGFR, donde afatanib ha mostrado un incremento de la supervivencia libre de progresión frente a la quimioterapia.

Las reacciones adversas que se han manifestado con mayor frecuencia han sido: diarrea, estomatitis, erupciones, dermatitis acneiforme, prurito, piel seca, paroniquia, disminución del apetito y epistaxis.

\section{ALOGLIPTINA (VIPIDIA $\left.{ }^{\circledR}\right)^{7,9}$}

La alogliptina es un inhibidor de la dipeptidil peptidasa 4 (DPP 4), enzima responsable de la inactivación de las incretinas GLP-1 (péptido similar al glucagón 1) y del GIP (polipéptido insulinotrópico dependiente de la glucosa). Esta inhibición produce un incremento plasmático de la incretina, con el consiguiente incremento en la liberación de insulina y disminución de la liberación de glucagón.

La indicación aprobada para este medicamento es el tratamiento de pacientes adultos con diabetes mellitus tipo 2 , para mejorar el control glucémico en combinación con otros medicamentos hipoglucemiantes, incluyendo la insulina, 
cuando éstos, junto con la dieta y el ejercicio, no proporcionen un control glucémico adecuado.

Los efectos adversos más frecuentes observados en la fase de investigación clínica fueron: infección del tracto respiratorio superior, nasofaringitis, cefalea, dolor abdominal, reflujo gastroesofágico, prurito y erupción cutánea.

\section{ALOGLIPTINA / METFORMINA ( VIPDO-MET® $)^{7,10,11}$}

Combinación de dos principios activos hipoglucemiantes orales, la alogliptina y la metformina. La alogliptina ha sido previamente descrita en el epígrafe 3.

La metformina es una biguanida con efecto antihiperglucemiante que disminuye la glucemia basal y pospandrial. Al no estimular la secreción de insulina no produce cuadros de hipoglucemia. Su mecanismo de acción no está perfectamente definido, se cree que actúa a través de varios mecanismos: disminución de la producción hepática de glucosa, disminución de la absorción intestinal de glucosa y mejorando la sensibilidad a la insulina mediante el aumento de la captación y utilización de la glucosa periférica.

Este nuevo medicamento se ha autorizado en base a los estudios realizados sobre pacientes adultos con diabetes mellitus tipo 2, en tratamiento con metformina que no consiguen un adecuado control glucémico. Muestran que en dosis de 12,5 o $25 \mathrm{mg}$, una vez al día, asociado a 500 o 750 $\mathrm{mg}$ de metformina, se consigue una mejor respuesta según demuestra la evolución de la HbA1c en un periodo de 12 semanas.

La indicación aprobada para Vidomet es el tratamiento de la diabetes mellitus tipo 2 en pacientes adultos:

- En pacientes adultos, como adyuvante de ejercicio y dieta, para mejorar el control glucémico inadecuadamente controlado con las dosis máximas toleradas de metformina en monoterapia.

- En combinación con pioglitazona (terapia de combinación triple) como adyuvante de ejercicio y dieta, en pacientes adultos inadecuadamente controlados con la dosis máxima tolerada de metformina y pioglitazona.

- En combinación con insulina (terapia de combinación triple) como adyuvante de ejercicio y dieta, en pacientes adultos cuando la insulina en dosis estable y la metformina sola no proporcionan un control glucémico adecuado.

Las reacciones adversas más frecuentes observadas en los ensayos clínicos fueron nasofaringitis, infección del tracto respiratorio superior, cefalea, gastroenteritis, dolor abdominal, diarrea, vómitos, gastritis, reflujo gastroesofágico, prurito y erupción cutánea.

\section{ALOGLIPTINA / PIOGLITAZONA (INCRE-SYNC®) $)^{7,12-14}$}

Combinación de dos principios activos hipoglucemiantes, la alogliptina y la pioglitazona. La alogliptina ha sido pre- viamente descrita en el epígrafe 3. La pioglitazona es una tiazolidindiona que actúa a través de la activación de un receptor nuclear específico (receptor gamma activado por un proliferador de peroxisoma), que conduce a un aumento de la sensibilidad a la insulina de las células hepáticas, adiposas y musculoesqueléticas.

Este medicamento está indicado en el tratamiento como segunda o tercera línea de la diabetes mellitus tipo 2 en pacientes adultos. Se administrará siempre como complemento a la dieta y ejercicio, para mejorar el control glucémico, especialmente en pacientes con sobrepeso, en los que no se alcanza un control adecuado con la pioglitazona sola y para los que la metfornina no puede administrarse, bien por contraindicación o por intolerancia. También está indicado en combinación con metformina (terapia triple) en aquellos pacientes en los que no se alcanza un adecuado control glucémico con la dosis máxima tolerada de metformina y pioglitazona.

Existen distintos estudios clínicos que avalan la autorización de este tratamiento. En pacientes en tratamiento con metformina, con la glucemia mal controlada, se muestra eficaz en el control de la glucemia con disminuciones de los niveles de $\mathrm{HbA1c}$, siendo bien tolerado el tratamiento.

Una vez iniciado el tratamiento con Incresync $($ se debe controlar el tratamiento durante 3 a 6 meses, para evaluar la respuesta del paciente (por ejemplo reducción en HbA1c). En los pacientes que no muestren una respuesta adecuada se debe interrumpir el tratamiento.

Las reacciones adversas más frecuentes presentadas en los estudios clínicos realizados, de la alogliptina como tratamiento adicional a la pioglitazona son infecciones del tracto respiratorio superior, sinusitis, cefalea, náuseas, dispepsia, dolor abdominal, prurito, mialgia, edema periférico y aumento de peso. La piogliltazona puede producir retención de líquidos, que puede provocar o exacerbar una insuficiencia cardiaca. En distintos estudios epidemiológicos sugieren un aumento del riesgo de cáncer de vejiga en los pacientes diabéticos tratados con pioglitazona.

\section{CÉLULAS MONONUCLEARES AUTÓLOGAS DE SANGRE PERIFÉRICA ACTIVADAS CON PAP-GM-CSF (SIPULEUCEL-T) (PROVENGE®) $)^{2,15}$}

El principio activo de este medicamento consiste en células mononucleares autólogas de sangre periférica activadas con fosfatasa ácida prostática, fusionadas con el factor estimulante de colonias de granulocito-macrófago (sipuleucel-T). El mecanismo de acción de este principio activo es la inducción de una respuesta inmune específica frente a la fosfatasa ácida prostática (PAP), siendo éste un antígeno expresado en la mayoría de los tumores prostáticos.

La indicación autorizada para este medicamento es el tratamiento de pacientes adultos con cáncer de próstata metastásico (no visceral), resistente a la castración, asintomático o mínimamente sintomático, en el que no está clínicamente indicada la quimioterapia. 
Los ensayos clínicos realizados para su autorización, han demostrado mejoría en la supervivencia de los pacientes en comparación con placebo.

Durante la fase de investigación clínica Provenge ha sido en general bien tolerado. Las reacciones adversas que se han observado con mayor frecuencia han sido escalofríos, fatiga, fiebre, náuseas, artralgia, dolor de cabeza y vómitos.

\section{COBICISTAT ( TYBOST $®)^{7,16}$}

El principio activo cobicistat es un agente potenciador farmacocinético de agentes antirretrovirales. Los ensayos clínicos realizados con este principio activo han mostrado capacidad para inhibir el metabolismo mediado por el CYP3A aumentando la concentración plasmática de aquellos fármacos que son metabolizados a través de esta ruta metabólica.

Tybost está indicado como potenciador farmacocinético de Atazanavir 300 mg o de Darunavir 800 mg una vez al día, como parte de la terapia de combinación antirretroviral en adultos infectados por el virus VIH. Estos dos antirretrovirales presentan una biodisponibilidad oral limitada y un rápido metabolismo por el CYP3A. La administración conjunta con Tybost la vida media de esos antirretrovirales.

Los efectos secundarios manifestados con mayor frecuencia en los ensayos clínicos están asociados con niveles elevados de bilirrubina.

\section{DABRAFENIB (TAFINLAR® ${ }^{2,17,18}$}

El principio activo autorizado en este medicamento, el dabrafenib, es un inhibidor de la proteína kinasa (L01XE23) que inhibe las kinasas BRAF con mutaciones activadas en el codón 600 .

Está indicado para el tratamiento como monoterapia del melanoma no resecable o metastásico con la mutación BRAF V600, en pacientes adultos.

Los ensayos clínicos llevados a cabo para su comercialización han demostrado eficacia y seguridad en su uso. En comparación con dacarbazina, dabrafenib demostró un incremento del tiempo de supervivencia libre de progresión de la enfermedad, es decir, retrasa la progresión de la enfermedad y mejora la supervivencia.

Los efectos secundarios que se han manifestado con mayor frecuencia durante la fase de investigación clínica han sido hiperqueratosis, dolor de cabeza, pirexia, artralgia, fatiga, náuseas, papiloma, alopecia, erupción cutánea y vómitos.

\section{DEFIBROTIDA (DEFITELIO® $)^{7,19}$}

El principio activo de este medicamento es la defibrotida, y no se conoce totalmente el mecanismo de acción del mismo. En los ensayos clínicos realizados con este principio activo, se ha mostrado que contribuye a la disolución de coágulos sanguíneos, existiendo indicios que indican que podría proteger las células que recubren los vasos sanguíneos.
En base a los estudios realizados, se ha autorizado con la indicación de tratamiento de la enfermedad veno-oclusiva hepática grave, también conocida como síndrome de obstrucción sinusoidal, que se puede producir en pacientes sometidos a trasplante de precursores hematopoyéticos. Está indicado tanto para adultos como para niños mayores de un mes de edad.

Defitelio ya fue designado como medicamento huérfano en el año 2004, y la actual autorización por parte del Comité Europeo de Evaluación de Medicamentos (CHMP) ha sido en circunstancias especiales, revisándose la misma de forma anual.

Las reacciones adversas encontradas con mayor frecuencia han sido hemorragias, incluyendo la hemorragia gastrointestinal, pulmonar y epistaxis, aunque no se limita sólo a éstas, hipotensión y coagulopatía.

\section{FENOFIBRATO/SIMVASTATINA $(C H O L I B \circledR)^{2,20}$}

Este medicamento está formado por la combinación de dos principios activos con función hipolipemiante, el fenofibrato y la sinvastatina:

- El fenofibrato es un agonista del receptor activado por proliferadores de peroxisomas tipo alfa, reduciendo los niveles tanto de las lipoproteínas de baja densidad (LDL) como las lipoproteínas de muy baja densidad (VLDL), aumenta las lipoproteínas de alta densidad (HDL) y reduce los triglicéridos.

- La simvastatina presenta su actividad hipolipemiante mediante la inhibición de la 3-hidroxi-3-metilglutarilcoenzima A (HMG-CoA) reductasa, reduciendo los niveles normales y elevados de colesterol y LDL.

La indicación de esta combinación es como tratamiento adyuvante a una adecuada dieta y ejercicio en pacientes adultos, con alto riesgo de sufrir enfermedad cardiovascular, que presentan dislipidemia mixta con el fin de disminuir los niveles plasmáticos de triglicéridos e incrementar los niveles de la fracción HDL del colesterol, cuando el paciente tiene controlado los fracción LDL del colesterol tras haber estado en tratamiento con sinvastatina en monoterapia.

Las reacciones adversas más frecuentes observadas en la fase de investigación clínica de este nuevo medicamento fueron: infecciones del tracto respiratorio superior, gastroenteritis y aumento de la cifra de plaquetas. También se han observado incremento en los niveles plasmáticos de alanina aminotransferasa y creatinina sanguínea.

\section{FIBRINÓGENO HUMANO/TROMBINA HUMANA $\left(\text { EVARREST }{ }^{\circledR}\right)^{2,21}$}

Los principios activos autorizados en este medicamento son el fibrinógeno humano y la trombina humana. Estas proteínas, de forma conjunta, van a ser las responsables de la formación del coagulo de sangre. Ambas proteínas se encuentran de forma deshidratada sobre una superficie de un material absorbible. Cuando ambas proteínas entran en con- 
tacto con la superficie de una herida sangrante, el fibrinógeno y la trombina se activan formando un coagulo que va a servir de ayuda al control del sangrado, favoreciendo el cierre de la herida.

El Comité de Medicamentos de Uso Humano, tras evaluar los estudios presentados que mostraban hemostasis en el sangrado de tejido blando incluido el control del sangrado del parénquima en pacientes sometidos a cirugía hepática, ha emitido un informe positivo a la comercialización de este medicamento.

El medicamento está indicado para su utilización en pacientes adultos como adyuvante al manejo de la hemostasis en el lecho quirúrgico cuando las técnicas quirúrgicas estándar no son suficientes.

Dentro de los efectos adversos manifestados con mayor frecuencia en la fase de evaluación clínica, se han descrito: hemorragia e incremento del fibrinógeno. Además de estas reacciones adversas, se han descrito con menor frecuencia, pero mayor gravedad, aspiración y embolia pulmonar.

\section{FILGASTRIM $\left(\text { GASTROFIL }{ }^{\circledR}\right)^{7,22}$}

El principio activo filgastrim es un medicamento inmunoestimulante, que actúa regulando la producción y liberación de neutrófilos funcionales de la médula ósea.

El Gastrofil es un medicamento biológico similar (biosimilar) a Neupogen ${ }^{\circledR}$, que es el producto de referencia autorizado en la UE, mostrando este medicamento biosimilar en los estudios de calidad, seguridad y perfil de eficacia unos resultados comparables a Neupogen $\AA$.

Se ha aprobado su utilización en terapéutica en las mismas indicaciones que Neupogen $\AA$.

Los efectos adversos que se han producido con mayor frecuencia tras la administración de filgastrim, en los ensayos en pacientes oncológicos han sido incrementos plasmáticos de ácido úrico, lactato deshidrogenasa, gamma-glutamil transferasa y fosfatasa alcalina, disminución del apetito, dolor orofaríngeo, tos, disnea, náuseas, vómitos, alopecia, erupción cutánea, dolor óseo, torácico y musculoesquelético, astenia, fatiga e inflamación de mucosas. Tras la administración en tratamientos para la movilización de células progenitoras de sangre periférica en pacientes normales se han observado dolor musculoesquelético, cefalea y leucocitosis y trombocitopenia en el 41 y $35 \%$ de los donantes normales. En pacientes con neutropenia crónica grave se han manifestado dolor óseo y musculoesquelético, cefaleas y diarrea. También se han manifestado casos de esplenomegalia en estos pacientes, así como en pacientes con infección de VIH en tratamiento con filgastrim.

\section{FOLITROPINA ALFA $(O V A L E A P ®)^{7,23}$}

La folitropina alfa es una hormona recombinante humana estimulante del folículo [r-hFSH]. Es esencial para el crecimiento y maduración normal del gameto femenino y la inducción de la producción de esteroides gonadales norma- les. La producción endógena deficiente de FSH es una causa conocida de infertilidad.

Ovaelap es un medicamento biosimilar a Gonal-f $\mathrm{A}$, producto de referencia autorizado en la UE. Ovaleap ${ }^{\circledR}$ ha mostrado en los estudios realizados una calidad, seguridad y perfil de eficacia comparables con Gonal-f®.

Las indicaciones autorizadas para Ovaleap son las mismas que para Gonal-f®.

Las reacciones adversas más comunes aparecidas tras la administración de folitropina alfa son cefalea, quistes ováricos y reacciones locales en el lugar de inyección. Se han notificado casos de hiperestimulación ovárica leve / moderada. El síndrome de hiperestimulación ovárica grave es muy poco frecuente. Se han reportado algunos casos de tromboembolismo, asociado generalmente al síndrome de hiperestimulación grave.

\section{INDACATEROL / BROMURO DE GLICOPIRRONIO (ULTRIBO BREEZHALER®IXOLERMA BREEZHALER $\left.{ }^{\circledR}\right)^{7,24-26}$}

Combinación de dos principios activos para administración por vía inhalatoria, el indacaterol, un agente beta2-adrenégico, que produce una relajación de la musculatura lisa de las vías aéreas, y el bromuro de glicopirronio, agente anticolinérgico que produce un bloqueo de la acción broncoconstrictora de la acetilcolina en las células del músculo liso de las vías aéreas, produciendo broncodilatación.

Estos dos medicamentos están indicados como tratamiento broncodilatador de mantenimiento para aliviar los síntomas en pacientes adultos con enfermedad pulmonar obstructiva crónica (EPOC). Los ensayos clínicos realizados en este grupo de pacientes, demuestran que indacaterol proporciona un efecto broncodilatador prolongado, que alcanza 24 horas con una única administración diaria. En estudios en comparación con placebo, ha mostrado una buena tolerancia, y un buen mantenimiento de la broncodilatación acompañado de una mejoría en la situación clínica de los pacientes.

Las reacciones adversas observadas con mayor frecuencia son síntomas beta-adrenérgicos y anticolinérgicos relacionados con los componentes individuales de la combinación.

\section{INFLIXIMAB (INFLECTA®/REMSIMA $\left.{ }^{\circledR}\right)^{2,27,28}$}

El principio activo de estos dos nuevos medicamentos comercializados es el infliximab. Es un anticuerpo monoclonal quimérico humano-murino, que se une con alta afinidad tanto a la forma soluble como a la forma transmembrana del factor de necrosis tumoral alfa (TNF $\alpha)$, pero no se une a la linfotoxina $\alpha(\mathrm{TNF} \beta)$. De esta forma se consigue el efecto inmunosupresor deseado.

Ambos medicamentos, Inflecta ${ }^{\circledR}$ y Remsima ${ }^{\circledR}$, son medicamentos biológico similar (biosimilares) al medicamento de referencia, Remicade ${ }^{\circledR}$ (Infliximab), autorizado en la Unión Europea el 13 de agosto de 1999. Los estudios 
realizados han demostrado que ambos medicamentos presentan un perfil de calidad, seguridad y eficacia comparable al de Remicade.

Las indicaciones autorizadas para estos dos medicamen-

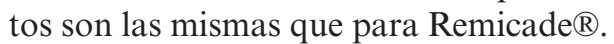

Dentro de las reacciones adversas que se han manifestado con mayor frecuencia en la fase de postcomercialización de infliximab, se han descrito reacciones de hipersensibilidad tras la infusión del fármaco, las infecciones respiratorias, incluida la tuberculosis e infecciones fúngicas invasivas.

\section{REGORAFENIB (STIVARGA® $)^{2,29,30}$}

El principio activo regorafenib es un inhibidor de la proteína kinasa que va a presentar actividad frente a las kinsasas involucradas a nivel del cáncer colorectal en la angiogénesis del tumor (VEGFR1,-2,-3, TIE2), en la oncogénesis (KIT, RET, RAF-1, BRAF, BRAF ${ }^{\mathrm{6} 600 \mathrm{E}}$ ) y en el microentorno del tumor (PDGFR, FGFR).

La especialidad Stivarga ${ }^{\circledR}$ ha sido autorizada para la indicación de tratamiento de pacientes adultos con cáncer colorectal metastásico, que hayan sido tratado previamente con las terapias disponibles, incluyendo fluoropirimidinas, terapias anti-VEGF y anti-EGFR, o bien que no puedan ser tratados con dichas terapias por no ser considerados candidatos a recibirlas.

En los ensayos clínicos realizados en pacientes con cáncer colorectal metastásico en los que ha existido progresión con las terapias estándar, regorafenib en comparación con placebo han demostrado un aumento de la supervivencia de 6,4 meses frente a los 5 meses del grupo control.

Como efectos adversos más frecuentes, se han observado: astenia/fatiga, falta de apetito y disminución de la ingesta alimentaria, reacciones cutáneas en manos y pies, diarrea, pérdida de peso, infecciones, hipertensión y disforia.

\section{BIBLIOGRAFÍA}

1. Committee for Medical Products for Human Use (CHMP) EMA 377379/2013 27 June 2013 Disponible en: http://www.ema.europa.eu/docs/en GB/document_library/Summary_of_opinion___Initial_authorisation/human/003718/ WC500144904.pdf (Consultado 10/10/13).

2. AgenciaEspañoladeMedicamentosyProductosSanitarios.Informemensualsobre Medicamentosde Uso Humanoy Productos Sanitarios Junio 2013. Disponibleen: http://www.aemps.gob.es/informa/informeMensual/2013/junio/informe-medicamentos.htm (Consultado 10/10/13).

3. The CAMMS223 Trial Investigators. Alemtuzumab vs. interferon beta la in early multiple sclerosis. N Engl J Med 2008; 359: 1786-801.

4. Coles AJ, twyman CL, et al. Alemtuzumab for patients with relapsing multiple sclerosis after disease-modifying therapy: a randomized controlled phase 3 trial. The lancet 2012; 380:1819-28.

5. Cohen JA, Coles AJ, et al. Alemtuzumab versus interferon beta la as first-line treatment for patients with relapsing-remitting multiple sclerosis: a randomized controlled phase 3 trial. The Lancet 2012; 380: 1829-39.

6. Committee for Medical Products for Human Use (CHMP) EMA 447957/2013 25 July 2013 Disponible en: http://www.ema.europa.eu/docs/en_GB/document library/Summary of opinion - Initial authorisation/human/002280/ WC500146640.pdf (Consultado 10/10/13)
7. AgenciaEspañoladeMedicamentosyProductosSanitarios. Informemensualsobre Medicamentos de Uso Humano y Productos Sanitarios Julio 2013. Disponible en: http://www.aemps.gob.es/informa/informeMensual/2013/julio/informe-medicamentos.htm (Consultado 10/10/13).

8. Hirsh V, Cadranel J, et al. Symptom and quality of life benefit of afatinib in advanced non-small-cell lung cancer patients previously treated with erlotinib or gefitinib: results of arandomized phase IIb/III trial (LUX-Lung 1). J Thorac Oncol 2013; 8(2):229-37.

9. Committee for Medical Products for Human Use (CHMP) EMA 229786/2013 25 July 2013 Disponible en: http://www.ema.europa.eu/docs/en_GB/document library/Summary of opinion - Initial authorisation/human/002182/ WC500146655.pdf (Consultado 10/10/13).

10. Committee for Medical Products for Human Use (CHMP) EMA 223982/2013 25 July 2013 Disponible en: http://www.ema.europa.eu/docs/en GB/document library/Summary of opinion - Initial authorisation/human/002654/ WC500146659.pdf (Consultado 10/10/13).

11. Seino Y, Miyata Y, Hiroi S, Hirayama M, Kaku K. Efficacy and safety of alogliptin added to metformin in Japanese patients with type 2 diabetes: a randomized, double-blind, placebo-controlled trial with an open-label, longterm extension study. Diabetes Obes Metab 2012; 14(10):927-36.

12. Committee for Medical Products for Human Use (CHMP) EMA 229752/2013 25 July 2013 Disponible en: http://www.ema.europa.eu/docs/en GB/document_library/Summary_of_opinion_-_Initial_authorisation/human/002178/ WC500146654.pdf (Consultado 10/10/13).

13. Pratley Re, Reusch JE, et al. Efficacy and safety of the dipeptidyl peptidase-4 inhibitor alogliptin added to pioglitazone in patients with type 2 diabetes: a randomized, double-blind, placebo-controlled study. Curr Med Res Opin 2009;25(10):2361-71.

14. DeFronzo RA, Burant CF, et al. Efficacy and tolerability of the DPP-4 inhibitor alogliptin combined with pioglitazone, in metformin-treated patients with type 2 diabetes. J Clin Endocrinol Metab 2012; 97(5):1615-22.

15. Committee for Medical Products for Human Use EMA/CHMP/363851/2013 27 June 2013 Disponible en: http://www.ema.europa.eu/docs/en GB/document_library/Summary_of_opinion_-_Initial_authorisation/human/002513/ WC500144872.pdf (Consultado 10/10/13).

16. Committee for Medical Products for Human Use EMA/CHMP/413499/2013 25 July 2013 Disponible en: http://www.ema.europa.eu/docs/en GB/document_library/Summary_of_opinion_-_Initial_authorisation/human/002572/ WC500146622.pdf (Consultado 10/10/13).

17. Committee for Medical Products for Human Use EMA/CHMP/339899/2013 27 June 2013 Disponible en: http://www.ema.europa.eu/docs/en_GB/document_library/Summary_of_opinion_-_Initial_authorisation/human/002604/ WC500144848.pdf (Consultado 10/10/13).

18. Hauschild A, Grob JJ, et al. Dabrafenib in BRAF-mutated metastasic melanoma: a multicentre, open-label, phase 3 randomized controlled trial. The Lancet 2012;380:358-65.

19. Committee for Medical Products for Human Use (CHMP) EMA 455080/2013 25 July 2013 Disponible en: http://www.ema.europa.eu/docs/en_GB/document_library/Summary_of_opinion_-_Initial_authorisation/human/002393/ WC500146653.pdf (Consultado 10/10/13).

20. Committee for Medical Products for Human Use EMA 380955/2013 25 July 2013 Disponible en: http://www.ema.europa.eu/docs/en_GB/document_library/Summary_of_opinion_-_Initial_authorisation/human/002559/ WC500144880.pdf (Consultado 10/10/13).

21. Committee for Medical Products for Human Use EMA/CHMP/385035/2013 27 June 2013 Disponible en: http://www.ema.europa.eu/docs/en_GB/document_library/Summary_of_opinion_-_Initial_authorisation/human/002515/ WC500144888.pdf (Consultado 10/10/13).

22. Committee for Medical Products for Human Use EMA/CHMP/304525/2013 25 July 2013 Disponible en: http://www.ema.europa.eu/docs/en_GB/document_library/Summary_of_opinion_-_Initial_authorisation/human/002150/ WC500146600.pdf (Consultado 10/10/13).

23. Committee for Medical Products for Human Use (CHMP) EMA/422789/2013 25 July 2013 Disponible en: http://www.ema.europa.eu/docs/en_GB/document_library/Summary_of_opinion_-_Initial_authorisation/human/002608/ WC500147019.pdf (Consultado 10/10/13).

24. Committee for Medical Products for Human Use EMA/CHMP/347765/2013 25 July 2013 Disponible en: http://www.ema.europa.eu/docs/en_GB/document_library/Summary_of_opinion__Initial_authorisation/human/002679/ WC500146589.pdf (Consultado 10/10/13). 


\section{Evaluación positiva de medicamentos: junio/julio 2013}

25. Committee for Medical Products for Human Use EMA/CHMP/457182/2013 25 July 2013 Disponible en: http://www.ema.europa.eu/docs/en_GB/document_library/Summary_of_opinion___Initial_authorisation/human/003755/ WC500146591.pdf (Consultado 10/10/13).

26. Chapman KR, Rennard SI, Dogra A, Owen R, Lassen C, Kramer B. Longterm safety and efficacy of indacaterol, a long-acting b2-agonist, in subjects with COPD: a randomized, placebo-controlled study. Chest 2011; 140:68-75.

27. Committee for Medical Products for Human Use EMA/CHMP/363689/2013 27 June 2013 Disponible en: http://www.ema.europa.eu/docs/en_GB/document_library/Summary_of_opinion_-_Initial_authorisation/human/002576/ WC500144832.pdf (Consultado 10/10/13).
28. Committee for Medical Products for Human Use EMA/CHMP/364710/2013 27 June 2013 Disponible en: http://www.ema.europa.eu/docs/en_GB/document_library/Summary_of_opinion_-_Initial_authorisation/human/002778/ WC500144831.pdf(Consultado 10/10/13).

29. Committee for Medical Products for Human Use (CHMP) EMA /358125/2013 27 June 2013 Disponible en: http://www.ema.europa.eu/docs/ en GB/document library/Summary of opinion - Initial authorisation/human/002573/WC500144844.pdf (Consultado 10/10/13).

30. Grothey A, Van Cutsem E, et al. Regorafenib monotherapy for previously treated metastatic colorectal cancer (CORRECT): an international, multicentre, randomized, placebo-controlled, phase 3 trial. Lancet 2013;381:303-12. 